\title{
Smart Subway Information Platform based on Internet of Things
}

\author{
Jun-Song Fu, Zhen-Jiang Zhang ${ }^{*}$, Xin-Lei Jin and Zi-Qi Hao \\ Department of Electronic and Information Engineering, Key Laboratory of \\ Communication and Information Systems, Beijing Municipal Commission of \\ Education, Beijing Jiaotong University, Beijing, 100044, China
}

\begin{abstract}
In the current urban rail transit system, the levels of informatization and integration are not high. In order to change the traditional pattern of rail transportation operation and management, we propose an intelligent information platform of subway system based on Internet of things. Depending on the sensor nodes deployed in various functional departments of rail transport and the RFID technology, this system transmits the collected data to the database server through the gateway node by deploying. After fuse the data from multi-sensor, the system possesses the data in wisdom subway information integrated platform uniformly. Simultaneously, this paper also proposes a completely covered algorithm in the monitoring areas as one of the key technologies applied in the information platform.
\end{abstract}

Key words: rail transport; The Internet of things; Information platform; Data fusion; Sensor deployment

\section{Introduction}

Boosted by the urbanization, the urban rail transport operation develops rapidly. But the traditional operation management mode cannot accommodate the needs of the rail transport operation development, due to the low levels of informatization and the integration. For the timely monitoring and security operations, in rail transportation operation network, environment information collection methods of informatization and integration are required to ensure rail transportation operation safety and efficient. On the one hand, the safe operation of urban rail transit requires the parameters along the route environment information within the normal range, such as temperature, humidity, smoke concentration and so no. The safety of rails and bridges, on the other hand, are also required to accept regular monitoring, such as the subsidence of the ground surface along track and horizontal displacement of the bridge. In order to maintain the environment of urban rail transport and avoid security incidents, taking the Internet of things technology as the core, integrating wireless sensor network technology and RFID technology, wisdom subway information platform of the subway system came into being [1]. 


\section{Hierarchical Functional Structure Design}

\subsection{The hierarchical structure of the information platform design}

Wisdom subway information platform design which is based on the Internet of things is shown in figure 1. The hierarchical structure of the system are composed with four layers named Internet of things sensing layer, base station transport layer, data processing layer and platform application layer respectively [2].

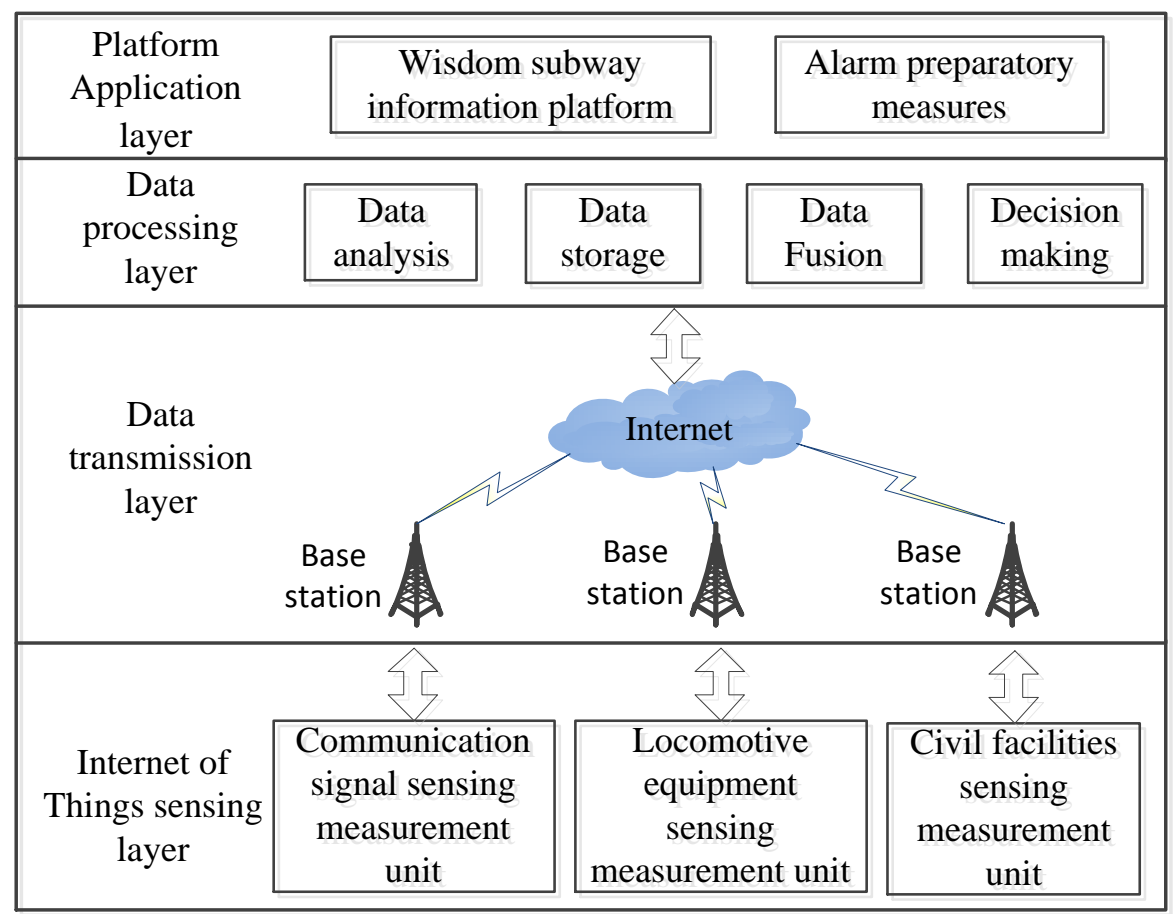

Figure 1. Platform structure chart

Internet of Things sensing layer mainly includes three modules which are communication signal sensing measurement unit, locomotive equipment sensing measurement unit and civil facilities sensing measurement unit. Its function is collecting information of rail transport monitoring area by the Internet of Things sensor device.

Data transmission layer mainly gathers the signal collected by the sensor nodes through the base station and sends them to the data processing layer.

Data processing layer analyzes and processes the data from data transmission layer, and stores the data in the server. Then the heterogeneous data is processed to data fusion. And after making decision, the data information is sent to the platform application layer [3].

Platform application layer is the monitoring center of the wisdom subway information platform. This layer can alarm automatically when the results from data processing layer beyond the predefined range. Thus the remote real-time monitoring can be truly achieved to ensure the rail transport operation safe and efficient. 


\subsection{The functional design of IOT (Internet of Things) sensing layer}

The three modules in the IOT perception layer can cover five main branches of rail transport sector which are mechanical and electrical company, the line company, JianAn Company, TongHao company and the vehicle equipment company [4].

The communication signal measuring unites deployed in the TongHao Company. The sensor nodes in the metro vehicle can maintain the signal communication between the different trains or between the ground and the train. Moreover, the sensor nodes are used to possess turnout operation automatically, which reduces the unreliability caused by manual operation.

Locomotive equipment sensing measurement units deployed in the mechanical and electrical company and vehicle equipment Company. In the mechanical and electrical company, we place the sensor nodes in the locomotive to monitor the speed to ensure traffic safety. Additionally, we can place dynamic gateway nodes to receive the aggregate information from the wireless sensors that are deployed between the rails, and then send data to the database of information platform when the trains come back. In the vehicle and equipment company, we can deploy sensor nodes to monitor the loss and the temperature of subway parts, as well as the connection among the carriages of train. If the joints of carriages accidentally parted, this system will feedback the information by sensors and the carriages will immediately brake. Moreover, we can give each subway train electronic tags with RFID technology and grasp the traveling information of the trains in the wisdom subway platform through the intelligent monitoring network to facilitate unified scheduling and the handling of emergency situations.

The civil engineering facilities sensing measurement unit is mainly laid on the line and construction-installation companies. In line companies, we can deploy wireless sensors in each subway station to monitor the temperature, humidity, smoke in the subway stations. If unexpected situations appear, it can achieve early warning. And for the management of subway passenger revenue, we can use RFID technology in the Internet of Things to realize intelligent ticketing. To be specific, people enter the subway by RIFD tag card and complete the payment. It can facilitate the management since the entire smart ticketing system is integrated in the wisdom subway information platform. In construction-installation companies, we deploy wireless sensors along the rail transit line and use sensor nodes of different types to monitor the surrounding environment of the rail lines. Especially the sensor nodes deployed around bridges and tunnels can effectively monitor the information about the subsidence of the surface of the earth and the displacement of bridges. Finally, the gateway node deployed in the locomotive receives the information above and returns to the wisdom subway information platform. This can help us to effectively monitor whether the situation of the railway lines is safety and ensure the normal and safe operation of the subway.

\section{Structural Design of Network Functions}

Figure 2 shows the structure of wisdom subway information platform that based on Internet of Things. It contains the node coverage area, the gateway node in WSN, data acquisition terminal, subway enterprise cloud and wisdom subway information platform. 


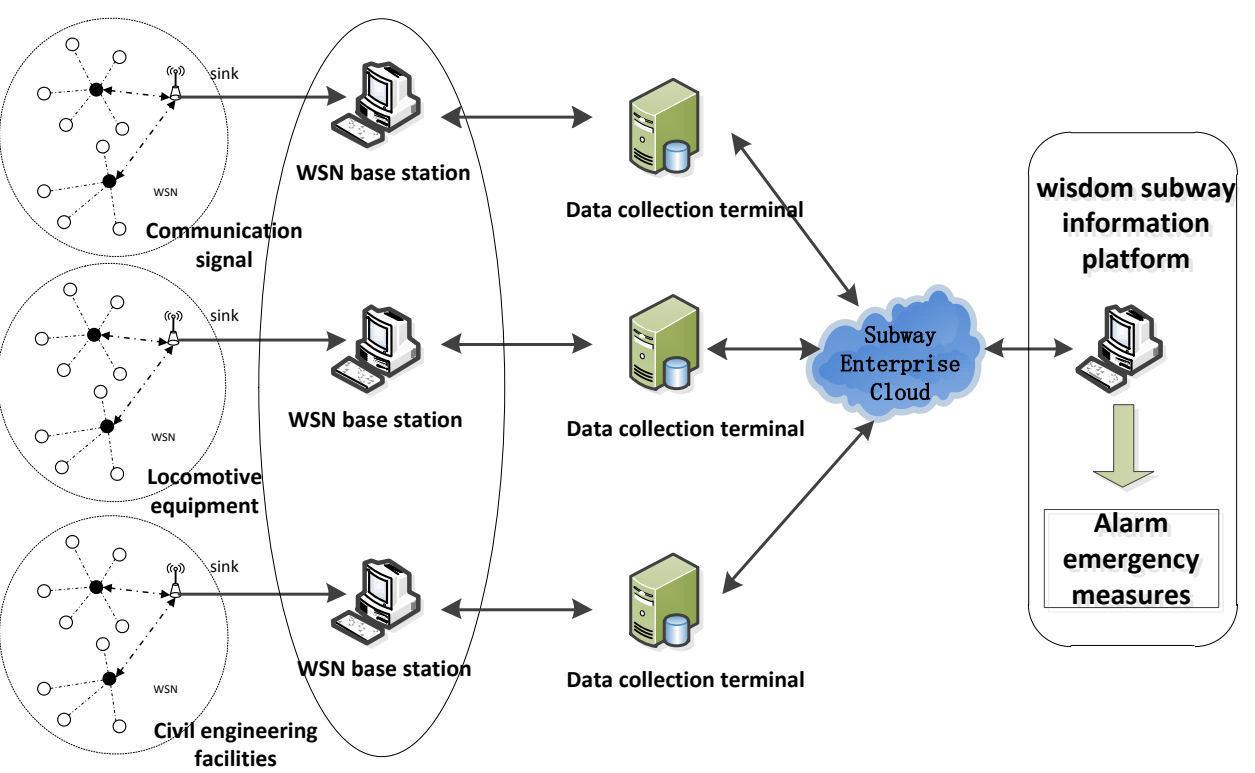

Figure 2. The structure of wisdom subway information platform

Node coverage area is mainly composed of wireless sensor networks (WSNs). WSNs consist of cluster tree network topology through sensor nodes, relay nodes and the sink node. A single node achieves sensing data of its area. Between nodes it achieves mutual communication and information transfer. The sink node is responsible for the formation of networks and acts as a gateway node to achieve the summary of information and sent to the database server.

Data collection terminals accept all the information from the base station. This collection of the information is the data that all sensor nodes monitored along the subway.

The wisdom subway information platform of the subway enterprise cloud is connected with the data collection terminal, and is responsible for monitoring as well as processing all data information along the subway to make assessment and scheduling for the hidden dangers or unexpected events. The functions include: data monitoring and analysis, node setting, the releasing of dynamic data to the Internet and alarm emergency measures etc. Under the situation that the monitoring platform sends out a warning but no response within the limited time, emergency measures will take emergency measures automatically to stop or slow down accidents in time. For example, there are fire alarms but no response in a limited time. It must start emergency measures such as closed fire doors, cut off the power equipment and so on.

\section{The Key Technologies of the System Function}

\subsection{Multi-sensor data fusion}

Much rail transportation equipment and infrastructure need to be monitored, and the failure of the appearance characteristics are also different. To achieve accurate early warning, we should take advantage of the different characteristics of a variety of sensors for all kinds of mechanical and electrical equipmentlocal conditions and data. You can get the consistency of interpretation and description of the measured object [5] to overcome the uncertainties and limitations of a single sensor, through the operation analysis in multi-sensor coordination between complementary and performance. 
Data fusion technology involves signal processing, feature extraction and reasoning. The multi-sensor data fusion technology applied in our platform is composed by these three parts, as shown in Figure 3. The interference and noise among the data can be filtered out in pre-processing model. Afterwards, process the feature extraction towards the target signal and then fuse the data based on the extracted feature signal. The final result can be output at last $[6]$.

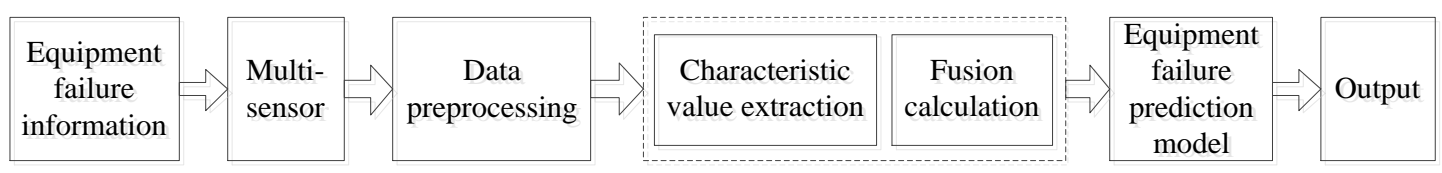

Figure 3. Multi-sensor system data fusion process

During the process of equipment failure recognition, we use the method of combining the data level fusion and decision level fusion [7]. Applying the first level (data) fusion, the data set can be transformed to the more accurate result, which is collected from the same kind of sensors deployed in the same zone, as shown in Figure 4.The fusion process which gives a more accurate measured value can be used to display the measured environment. The comparing between fusion result $S_{i}^{\prime}$ and pre-set threshold $T_{i}$ can effectively explain whether the monitoring project is failure.

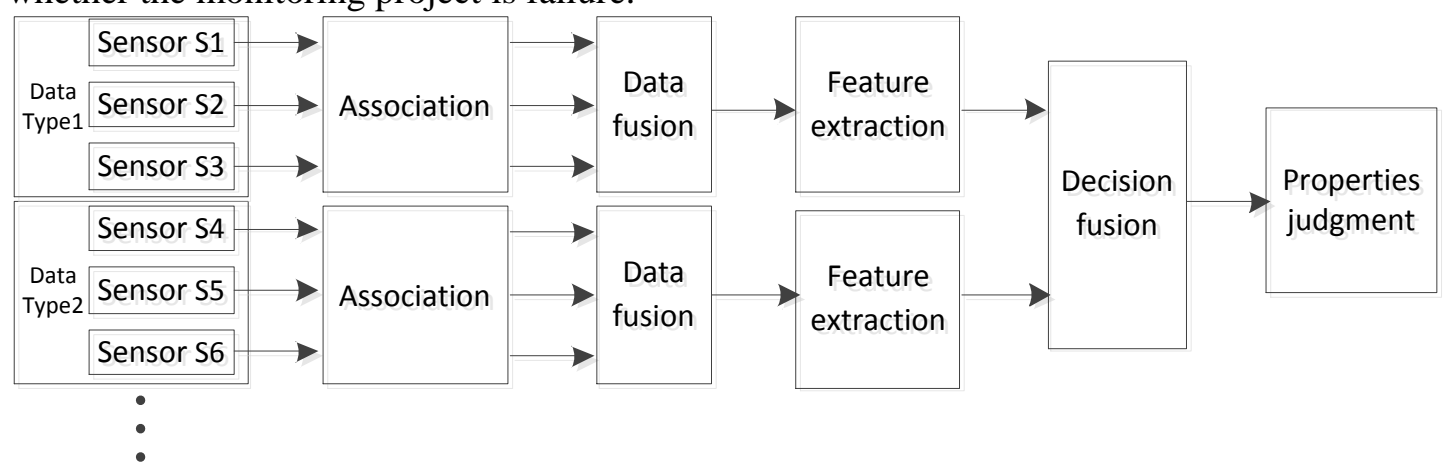

Figure 4. Multi-sensor data fusion structure diagram

At the same time, it is necessary for all monitoring data to be measured by an overall measurement in the decision-making judge in order to understand the overall urban rail transit system condition. Thus, the results from above data fusion should be fused again by decision-making level data fusion. The decision level fusion is the highest level of data fusion. After pretreating the data from the varieties of sensors, we can make the independent decisions to the target respectively. In order to get the final decision result which is consistent with the real status, we fused the independent decisions in the decision-making level.

The weighted evaluation method of decision-making data fusion is an effective way in most cases. Considering the degree of importance of the different types of observational data in the entire rail transit system by experts, we assign weights to the various types of data. We suppose there are $\mathrm{n}$ data fusion results at the same time on the same system level, the fusion results are: $S_{1}^{\prime}, S_{2}^{\prime}, \cdots, S_{n}^{\prime}$. In addition, there are weight values assigned by the expert $a_{i}(\mathrm{i}=1,2, \ldots, \mathrm{n})$, and shall meet the $\sum_{i=1}^{n} a_{i}=1$. Additionally, there is a threshold set for 
different types of data $T_{i}(\mathrm{i}=1,2, \ldots, \mathrm{n})$. The final evaluation coefficient can be obtained by weighted summation evaluation method as follows:

Comprehensive Evaluation coefficient

$$
\begin{aligned}
& =S_{1}^{\prime} / T_{1} \times a_{1}+S_{2}^{\prime} / T_{2} \times a_{2}+S_{3}^{\prime} / T_{3} \times a_{3}+\cdots+S_{n}^{\prime} / T_{n} \times a_{n} \quad \text { (Formula 1) } \\
& =\sum_{i=1}^{n}\left(S_{i}^{\prime} / T_{i} \times a_{i}\right)
\end{aligned}
$$

Among this, $S_{n}^{\prime} / T_{n}$ is the ratio of data fusion results to default expert's threshold. If the integration result is greater than the threshold, then the ratio is greater than 1 . When all the measured values do not exceed the preset threshold in all types of monitoring environment, the system obtained by a combination factor must be less than 1 . When the system combination factor is close to 1 , it is believable that the rail transit system is most likely to fail. In this case, decisions should be made to troubleshoot problems in the whole system.

\subsection{The sensor node coverage strategy along the rail traffic}

We need to collect the useful information of monitoring areas by deploying sensors. For example, in order to get the numerical information of the temperature, humidity, smog inside the subway station, we need to deploy the sensor nodes along the rail to achieve the area monitoring. When designing the coverage strategy of sensor nodes, the following issues should be considered: First, in order to ensure the coverage and enhance the quality of service of the network, we should try to avoid the coverage holes (religions that do not covered by any sensor). Second, minimize the sensor cost when the coverage rate meets the requirement. Using a mixed WSN which include both static and mobile nodes can achieve a balance between sensor cost and coverage. In our platform, we propose to deploy both mobile and static sensors in the rail transit monitoring area. The coverage holes can be detected by topology of network after initial deployment, then apply the mobility of mobile sensors to heal the coverage holes in the monitoring area.

We proposed a Voronoi based nibble coverage algorithm (VNCA) to cover the monitoring area. We make the following assumptions in order to simulate VNCA. First, each sensor can determine its position by the existing positioning technology. Second, the sensing model of a sensor is the sensing disk model where a sensor can cover a disk centered at itself with a radius equal to a fixed sensing range [8].

We use the method proposed in [9] to detect the coverage hole of a sensor. Assuming that each static node only need one mobile node to heal the coverage hole around its Voronoi cell. If there exists a hole, the static node chooses the farthest Voronoi vertex as the possible target location of the coming mobile node. As illustrated in Figure 5, $V_{1}$ is the farthest Voronoi vertex of sensor $S_{1}$, so we choose $V_{1}$ as the possible target location of the mobile sensor nodes. When two or more static nodes shares the same farthest Voronoi vertex, we assume only one mobile node is needed to heal the hole around the static nodes. The hole size is calculated by $\pi\left(d-R_{s}\right)^{2} . d \geq R_{s}$ approximately, where $d$ is the distance between the static node and its farthest Voronoi vertex, $R_{s}$ is the unique sensing range of all nodes. 


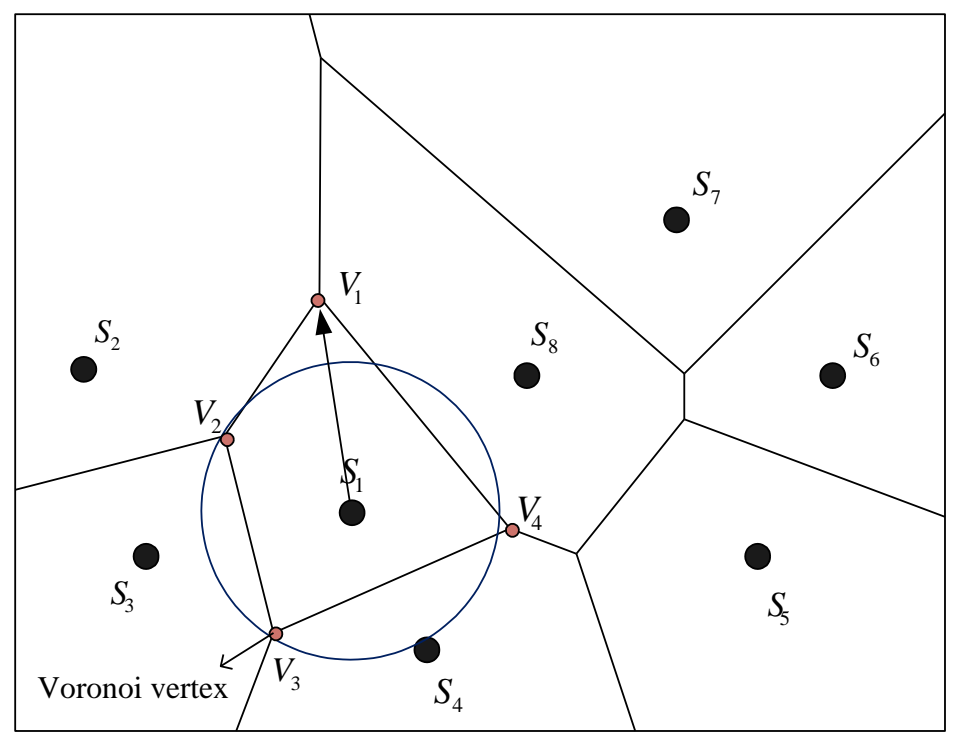

Figure 5. illustration of using Voronoi diagram to detect a coverage hole

The steps of our Voronoi based nibble coverage algorithm (VNCA) are as follows:

1. Construct Voronoi diagram with all the static nodes of the sensing region, each static node detect the hole around itself and calculate the hole size according to its Voronoi polygon.

2. Sort the detected holes from big to small and select the hole whose size is closest to the coverage area of a single node, then the centre of the hole is the target location of the coming mobile node in this round.

3. Placing a virtual static node in the target location determined in step 2, then turn to step 1.

4. Repeat step 1, 2, 3, until the number of target locations is equal to the mobile nodes number, or the coverage performance is up to grade.

5. Use appropriate matching algorithm to match the mobile nodes with target locations, thus to determine the target location of each mobile node.

The VNCA can be implemented by the following method. Initially, all nodes send their position coordinates to the sink node. And then the sink nodes collect all the position information and send them to wisdom subway information platform. VNCA is run on the information platform and the calculated target locations of each sensor node are sent to the sink node. Then the sink node feeds back the results to each mobile node. Finally, mobile nodes perform physical movement to heal the coverage holes.

We simulate by MATLAB to verify the coverage performance of VNCA. The strip area along the railway can be divided into multiple rectangular areas, and we take one of the rectangular regions into account to verify the coverage algorithm. Assuming a sensing area of 50 (units) x 50 (units) square, and the unified sensing range of the sensors is 5 units. We deploy 28 static sensors in the sensing area. Figure 6 is the simulation results of randomly deploy 28 static nodes. The Voronoi vertex marked with a ' $X$ ' is the coverage holes detected by each Voronoi cell of the sensor. 


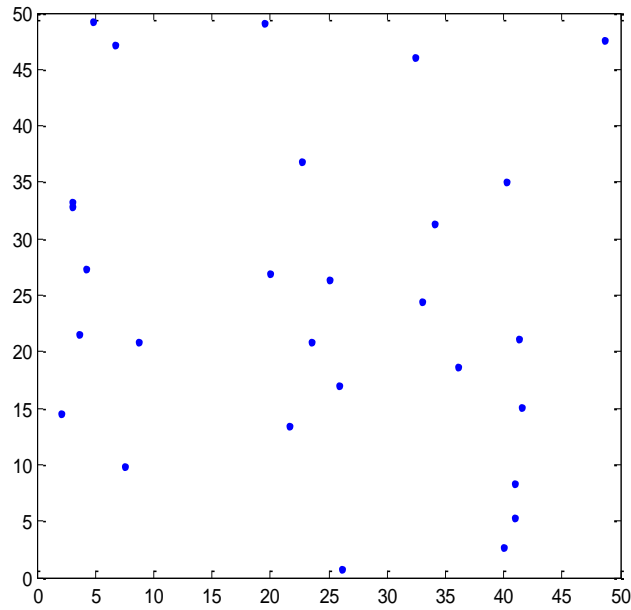

Figure 6. Deploying 28 static nodes

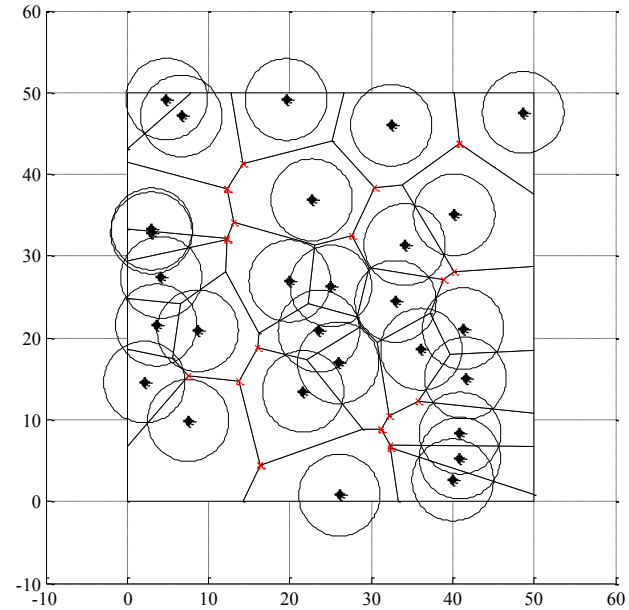

Figure 7. Hole detecting

Figure 8 shows coverage performance of VNCA after 19 rounds. The centre of red dashed circle is the target location of mobile sensor in current round. According to the simulation results, we came to the coverage rate of round $10,15,19$ is $78.22 \%, 84.23 \%, 87.10 \%$ respectively.

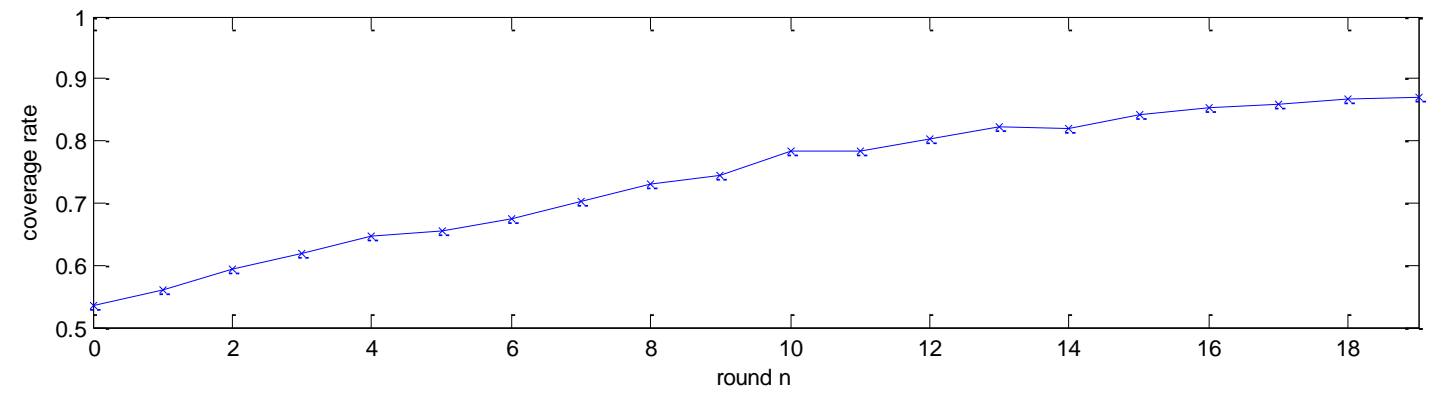

Figure 8. Coverage performance of VNCA after 19 rounds

Figure 9 is the comparison between immediate coverage and VNCA when they have the same mobile sensors number. The immediate coverage is the strategy that migrate the mobile nodes to the holes detected in Figure 7 directly. Calculated results show that immediate coverage needs 19 sensors for gaining the coverage of $77.8 \%$, and VNCA gain coverage of $87.10 \%$ after 19 rounds, which has increased $9.3 \%$. Actually, in the $10^{\text {th }}$ round, the VNCA has already achieved a coverage of $78.2 \%$, which saved 9 mobile sensors comparing with the immediate coverage under the same coverage rate. 

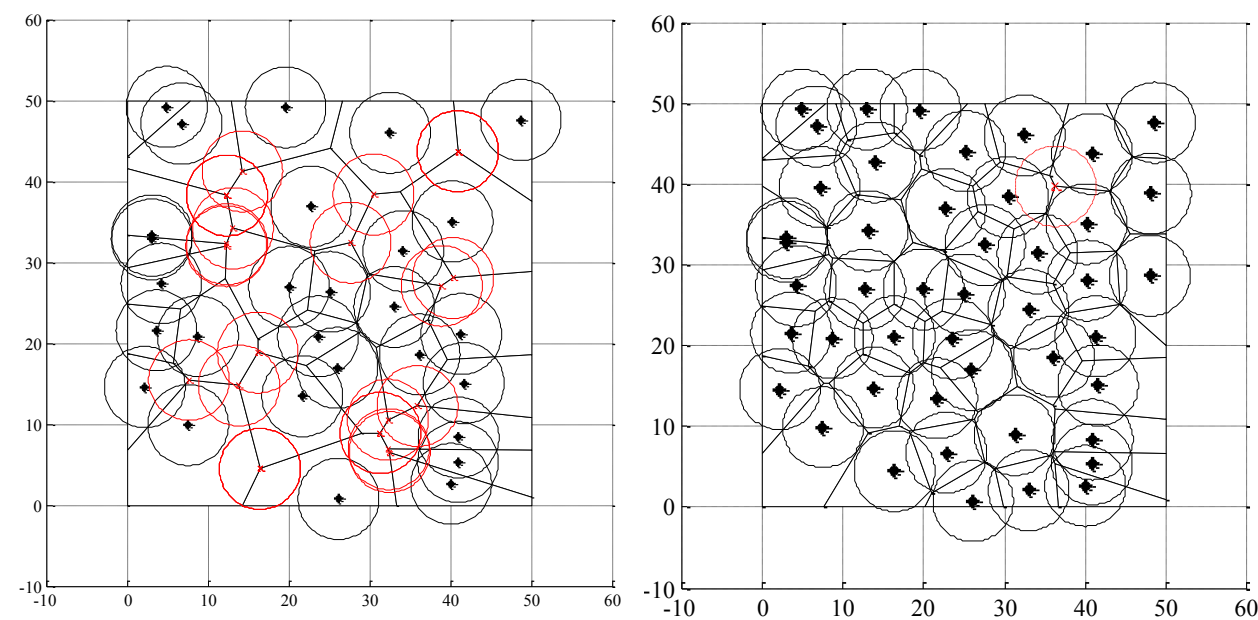

Figure 9. Coverage performance of VNCA after 19 rounds

\section{Conclusion}

This article describes a set of wisdom subway information platform system, which combines with the management of digital technology to the entire Metro departments of information and intelligence management. The system structure includes the coverage area of base station node, the base station gateway node, the database server and the terminal monitoring platform. This information system also adopts the VNCA as the sensors deployment strategy to achieve high coverage rate with the fewer sensors. In order to achieve accurate warning of failure, the multi-sensor data fusion technology is used to overcome uncertainties and limitations of a single sensor node. The information collected by nodes will sent to the wisdom subway platform through the gateway node aggregation, which could achieve the the safe and efficient operation of the rail transportation unified monitoring.

\section{Acknowledgements}

This research is supported by National Natural Science Foundation of China under Grant 61071076, the National High-tech Research and Development Plans (863 Program) under Grant 2011AA010104-2, the Beijing Municipal Natural Science Foundation under Grant 4132057.

\section{References}

[1] X. -d. Chen and Y. Bin, "Smart rail transportation-in-depth sensing and perceiving", Journal of Computer Application, vol. 32, no. 5, (2012), pp. 1196-1198.

[2] X. Li, R. Lu, X. Liang, X. Shen, J. Chen and X. Lin, "Smart community: an internet of things application", Communications Magazine, IEEE, vol. 49, no. 11, (2011) November, pp. 68-75.

[3] J. Lin, J. Yin and J. Cheng, "Research on the Multi-Sensor Data Fusion Technology for Network Security", Computer Engineering and Science, vol. 32, no. 6, (2010).

[4] T. Fuxing, "Introduction to Urban Rail Transit", China Railway Press, Beijing, (2013).

[5] X. Liao, M. Qiu and H. Mai, "The Study on Data Fusion Algorithms of Multi-Sensor Based on Parameter-Estimation”, Chinese Journal of Sensors and Actuators, vol. 20, no. 1, (2007), pp. 193-197. 
International Journal of Hybrid Information Technology

Vol.6, No.6 (2013)

[6] W. Jiewen, Y. Jianguo and Z. Shimin, "Application of Intelligent Early-warning Technology in Monitoring System of Metro Station Equipment”, Urban Rapid Rail Transit, vol. 21, no. 3, (2008).

[7] K. Bahador, K. Alaa and O. Fakhreddine, "Multisensor data fusion:A review of the state-of-the-art", Information Fusion, vol. 14, (2013), pp. 28-44.

[8] B. Wang, H. B. Lim and D. Ma, "A Survey of Movement Strategies for Improving Network Coverage in Wir eless Sensor Networks", Computer Communications (Elsevier), vol. 32, no. 13-14, (2009).

[9] G. Wang, G. Cao, P. Berman and T. F. L. Porta, "Bidding protocols for deploying mobile sensors", IEEE Transactions on Mobile Computing, vol. 6, no. 5, (2007), pp. 515-528. 